\title{
Noradrenaline and Dopamine Elevations in the Rat Prefrontal Cortex in Spatial Working Memory
}

\author{
Zvani L. Rossetti ${ }^{1,2}$ and Sonia Carboni ${ }^{1}$ \\ ${ }^{1}$ Department of Neuroscience and ${ }^{2}$ Consiglio Nazionale delle Ricerche Institute of Neuroscience, University of Cagliari, 09042 Monserrato, Italy
}

The role of prefrontal cortical dopamine (DA) in the modulation of working memory functions is well documented, but substantial evidence indicates that the locus ceruleus noradrenergic system also modulates working memory via actions within the prefrontal cortex (PFC). This study shows that PFC noradrenaline (NA) and DA dialysate levels phasically increase when rats perform correctly in a delayed alternation task in a T-maze, a test of spatial working memory. However, NA levels were markedly enhanced in animals trained to alternate compared with rats that acquired the spatial information about the location of food in the maze but were untrained to make a choice to obtain the reward. In contrast, PFC DA elevations occurred independently of whether the animal had acquired the trial-specific information for correct task execution. The contribution of anticipatory responses to catecholamine efflux was also evaluated by exposing rats to an environment signaling the presence of the reward in the successive alternation task. No conditioned NA efflux was observed in either group. In contrast, in both groups, DA efflux increased in the anticipatory phase of the test to the same levels of those reached during the task. These data provide the first direct evidence for a selective activation of PFC NA transmission during a spatial working memory task. We propose that, in the working memory task, DA is primarily associated with reward expectancy, whereas NA is involved in the active maintenance of the information about a goal and the rules to achieve it.

Key words: noradrenaline; dopamine; microdialysis; T-maze; selective attention; reward prediction

\section{Introduction}

The locus ceruleus (LC)-noradrenergic system exerts a modulatory influence on working memory functions subserved by the prefrontal cortex (PFC) (Arnsten, 2001). $\alpha$-2 Noradrenergic receptor agonists selectively improve reduced working memory performance in aged monkeys (Arnsten et al., 1988) and rats (Tanila et al., 1996) or in animals with pharmacological or anatomical lesions of the ascending catecholamine pathway (Arnsten and Goldman-Rakic, 1985; Cai et al., 1993). Conversely, noradrenergic antagonists reduce delay-dependent neuronal activity in the monkey PFC (Sawaguchi, 1998; Li et al., 1999), and, when injected locally in the PFC, they impair working memory performance (Li and Mei, 1994). Current accounts of attentional functions propose an important role for the PFC (Desimone and Duncan, 1995), and studies of working memory in primates have shown selective responses of PFC neurons to behaviorally relevant spatial information (Rainer et al., 1998). Activation of the LC-noradrenergic system is associated with the increased accuracy of response to task-relevant stimuli (Aston-Jones et al., 2000; Berridge and Waterhouse, 2003). However, it is not known

Received July 26, 2004; revised Dec. 23, 2004; accepted Dec. 26, 2004.

This work was supported by a grant (COFIN number 9805108207/2001) from the Ministry of University and Scientific Research (Z.L.R.). We are grateful to Dr. Paul B. Clarke for the thoughtful review of this manuscript, Dr. Liana Fattore for helpful comments, and Antonio Boi and Roberta Mannarino for assistance with behavioral testing.

Correspondence should be addressed to Zvani L. Rossetti, Department of Neuroscience, University of Cagliari, Cittadella Universitaria, 09042 Monserrato (CA), Italy. E-mail: rossetti@unica.it.

S. Carboni's present address: Serono Pharmaceutical Institute, 15 bis Chemin Des Mines, 1121 Geneva 20, Switzerland. E-mail: sonia.carboni@serono.com.

DOI:10.1523/JNEUROSCI.3038-04.2005

Copyright $\odot 2005$ Society for Neuroscience $\quad$ 0270-6474/05/252322-08\$15.00/0 whether the activity of the LC-noradrenergic system is selectively heightened during a working memory task. Thus, the first goal of this work was to test the hypothesis that the execution of a delayed alternation task in a T-maze, a test of spatial working memory sensitive to proper function of the PFC (Goldman-Rakic, 1987; Granon et al., 1994; Zahrt et al., 1997), is associated with elevations of noradrenaline (NA) levels in the PFC.

In the present study, NA dialysate levels in the PFC were compared with those of dopamine (DA) in animals performing the task. The primary role of prefrontal cortical DA in the modulation of working memory functions is well documented (Sawaguchi et al., 1988, 1990; Sawaguchi and Goldman-Rakic, 1991; Williams and Goldman-Rakic, 1995; Wang et al., 2004). Increased DA release in the PFC parallels the performance of a working memory task in primates at short delay intervals $(5 \mathrm{~s}$ ) (Watanabe et al., 1997). Also, in rats, the elevations of PFC DA parallel the accuracy of working memory performance, but they do so at surprisingly long delays (30 or $60 \mathrm{~min}$ ) (Phillips et al., 2004). In current tests of spatial working memory in rodents, animals are first trained to acquire a rule to locate a reward in a maze and are then tested for performance in making a correct choice; that is, they have to make predictions of the outcome. However, DA neuronal firing signals reward prediction (Schultz and Dickinson, 2000), and firing rate increases from the expected time of reward in proportion to the uncertainty of the outcome (Fiorillo et al., 2003). Therefore, the conditions of maximal activation of DA neurons in response to reward anticipation seem undistinguishable from the conditions of working memory tasks.

Thus, in the present task, we also investigated whether anticipatory responses to the reward presented during the working 
Alternation-untrained

Alternation-trained

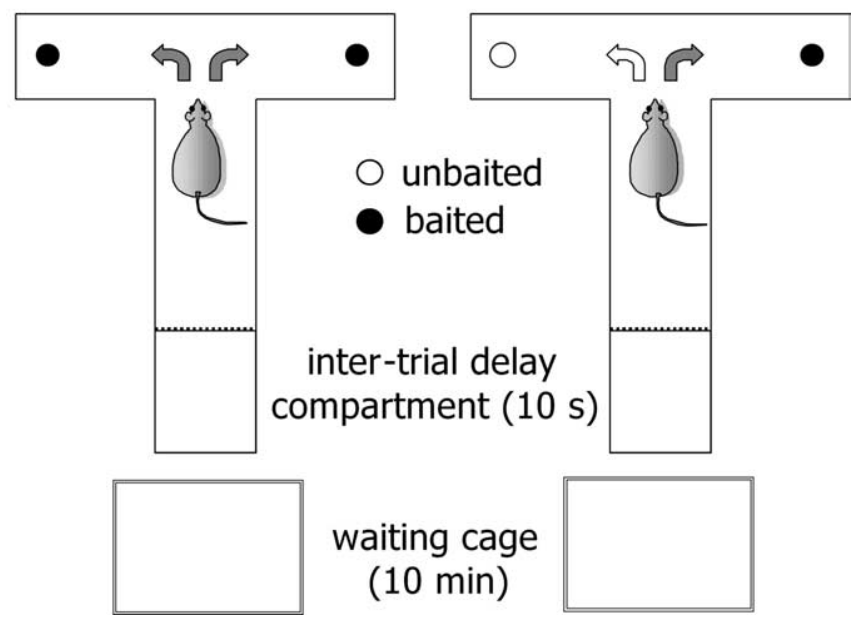

Figure 1. Schematic diagram of the delayed alternation task used in the present study. A group of rats was trained to search and locate the food reward in the T-maze with both arms always baited (Alternation-untrained), whereas a second group of animals was trained to alternate to locate the reward (Alternation-trained). During both training and testing sessions, all of the animals were first placed for $10 \mathrm{~min}$ in a cage adjacent to the T-maze (waiting cage) before visiting the maze. Testing in the maze was performed for 32 consecutive trials ( $10 \mathrm{~min}$ ). An interval of $10 \mathrm{~s}$ was introduced between the trials by placing the rats in the "intertrial delay compartment." For details, see Materials and Methods.

memory task contribute to prefrontal cortical NA and DA activation, as assessed by microdialysis. For this purpose, we compared animals trained to perform correctly in a delayed alternation task with animals that only acquired the spatial information about the location of the reward but were not trained to alternate.

\section{Materials and Methods}

Animals. Experiments were performed on adult male Sprague Dawley rats (weight, 250-300 g; Harlan-Nossan, Milan, Italy) in accordance with the European Communities Council Directive (86/609/ECC) for the care and use of laboratory animals and were approved by the Animal Care Committee of Cagliari University. Rats were housed individually at $22 \pm$ $2^{\circ} \mathrm{C}$ and $60 \%$ humidity on a $12 \mathrm{~h}$ light/dark inverted cycle (lights on at 7:00 P.M.). Home cages were located in a separate compartment of the testing room. Rats were food deprived for $23 \mathrm{~h} / \mathrm{d}$ to maintain $80 \%$ of normal body weight, with water available ad libitum.

Working memory task. Spatial delayed alternation performance was tested using a T-maze constructed from black polyvinyl, with the main alley $50 \mathrm{~cm}$ long, the side arm $40 \mathrm{~cm}$ long, walls $30 \mathrm{~cm}$ high, and alleys 15 $\mathrm{cm}$ wide (Fig. 1). Two removable doors close the side alleys. At the edge of each side arm, there is a small cup containing highly palatable food [one morsel of Fonzies (LU Snack Food, Donauwörth, Germany) weighing $\sim 60 \mathrm{mg}$ (Bassareo and Di Chiara, 1997)]. A holding compartment with a removable door was placed at the edge of the main alley for the intertrial interval (10 s). A waiting cage was placed adjacent to the T-maze. The entire T-maze was wiped with alcohol between different animals, and the choice point was wiped between different trials to suppress olfactory cues. Animals were handled for $3 \mathrm{~d}$ and then habituated to the maze with all of the doors open for $10 \mathrm{~min}$ for $5 \mathrm{~d}$. Animals were then food restricted, and each animal received $\sim 15 \mathrm{~g}$ of food per day immediately after the completion of the behavioral testing and thus maintained at $80 \%$ of their free-feeding weight throughout the experiment. The adaptation process continued by first placing the animal in the waiting cage for $10 \mathrm{~min}$ and then for $5 \mathrm{~min}$ in each side arm with food in the cup. The animal was then placed in the other arm, which also contained the food, for $5 \mathrm{~min}$. Subsequently, the actual alternation was started. Each rat received 12 trials per session, in two sessions per day (at 9:00 A.M. and 2:00 P.M.). Before the task, the animal was placed in the waiting cage for
$10 \mathrm{~min}$. On the first trial, animals were rewarded for entering either arm. Thereafter, during the next 11 trials, animals were rewarded only if they entered in the maze arm that was not chosen previously. Once the animal entered either arm, the door was closed, and the animal was removed and put in the holding compartment for a 10 s intertrial interval. This training continued until a criterion of 9 correct choices of 11 (82\%) on two consecutive sessions ( $5 \mathrm{~h}$ apart) was achieved. Animals that did not reach this criterion within 20 sessions were discarded. Animals were then tested in the maze for $2 \mathrm{~d}$ for correct performance during a continuous $10 \mathrm{~min}$ session (average, $32 \pm 1.2$ trials). The $10 \mathrm{~min}$ time interval was chosen to allow the collection of sufficient sample volume for catecholamine analysis. All of the alternation-trained animals $(n=9)$ that reached the criterion with 11 trials $(\sim 3-4 \mathrm{~min})$ did so also when the testing time was extended to $10 \mathrm{~min}$. A second group of animals $(n=9)$ underwent the same adaptation procedure described above for the alternation-trained rats, except that rats visited the maze with both arms always baited. Thus, this group (alternation-untrained group) acquired the spatial information for obtaining the reward but was untrained to alternate in the maze. As a control for the effect of conditioning on PFC catecholamine levels, two more groups of animals were used ( $n=5$ for each group): one was trained and the other untrained to alternate, respectively, as described above, except that, during the acquisition phase, a randomly variable interval of 10,15 , or $30 \mathrm{~min}$ was introduced between the $10 \mathrm{~min}$ waiting cage period and the testing in the maze (pseudoconditioning groups). On the day of the experiment, these groups of pseudoconditioned rats were tested in the maze immediately after the $10 \mathrm{~min}$ period spent in the waiting cage, as the conditioned rats. Animals were run at a random order regardless of the allocation group. All of the experiments were performed in a room with black walls under a dim light.

Surgery and microdialysis. After training, rats underwent stereotaxic implantation of chronic guide cannulas. Surgery was performed under chloral hydrate ( $400 \mathrm{mg} / \mathrm{kg}$, i.p.) anesthesia. Guide cannulas were of $20 \mathrm{ga}$ stainless steel directed to the cortex. Coordinates (of the cannula tip) (Paxinos and Watson, 1986), in millimeters from bregma, are as follows: anteroposterior, 3.2-3.7; mediolateral, \pm 0.5 ; dorsoventral, -1.2 . Cannulas (Plastics One, Roanoke, VA) were held in place with dental cement. A sterile stylette was screwed into place to keep the cannula patent. After surgery, animals were returned to their home cages. Approximately $6 \mathrm{~d}$ after surgery, animals were retested daily in the maze (10 min testing) until the performance criterion was resumed. At this time, concentric probes were inserted into the guide cannulas in the awake animal to -5.5 $\mathrm{mm}$ ventral to dura (infralimbic-cingulate PFC; exposed area, $4 \mathrm{~mm}$ ). After probe insertion, animals were connected to the perfusion pump via a balanced retention system attached to a liquid swivel and were left overnight in their home cage with probes being perfused at a flow rate of $0.5 \mu \mathrm{l} / \mathrm{min}$ with artificial CSF (aCSF) (in mM: $145 \mathrm{NaCl}, 1.2 \mathrm{CaCl}_{2}, 1.0$ $\mathrm{MgCl}_{2}$, and $2 \mathrm{KCl}$ ). On the day of the experiment, the flow rate was set to $1.8 \mu \mathrm{l} / \mathrm{min}$, and, after $1 \mathrm{~h}$ of stabilization, $10 \mathrm{~min}$ perfusate samples were collected for the determination of baseline levels of DA and NA (four samples within $10 \%$ variation). Thereafter, the animals were placed in the waiting cage for $10 \mathrm{~min}$ (one dialysate sample) and were then tested continuously in the maze for another $10 \mathrm{~min}$ interval. At the completion of the task, rats were moved back to their home cages, in which the collection of dialysate samples continued for an additional $60 \mathrm{~min}$.

Catecholamine assay. Extracellular concentrations of DA and NA were measured in $15 \mu \mathrm{l}$ samples. Monoamines were assayed by reverse-phase HPLC using a $50 \times 2.1 \mathrm{~mm}$ Restek (Bellefonte, PA) Pinnacle Amine, 5 $\mu \mathrm{m}$ particle size $\mathrm{C} 18$ column, with a mobile phase consisting of $50 \mathrm{~mm}$ phosphate buffer, $\mathrm{pH}$ 5.4, containing $1 \mathrm{~mm}$ EDTA, $200 \mu \mathrm{l} / \mathrm{l}$ tetraethyl amine, $50 \mathrm{mg} / \mathrm{l} \mathrm{SDS}$, and $8 \% \mathrm{v} / \mathrm{v}$ acetonitrile. Detection was accomplished in reduction mode with a Coulochem II (ESA, Chelmsford, MA) equipped with a model 1011 analytical cell set at 350 and $-180 \mathrm{mV}$ for the oxidation and reduction potential, respectively.

Histology. At the completion of the experiment, rats were killed by overdose of chloral hydrate and perfused intracardially with $5 \%$ paraformaldehyde, brains were excised and cut, and coronal sections (40 $\mu \mathrm{m})$ were stained with neutral red to localize the probe. In a randomly chosen subgroup of rats $(n=3), 3 \mathrm{~d}$ before being killed, probes were filled with the fluorescent retrograde tracer Fluorogold (FG) $(0.1 \%$ in aCSF) for 10 
min and then briefly perfused with aCSF to remove the dye. A microscope equipped with epifluorescence (BX60; Olympus Optical, Tokyo, Japan) was used to observe FG-positive cells in sections of PFC (Fig. 2b) or LC (Fig. 2c).

Data analysis. Statistical comparisons across the different experimental conditions were evaluated by a two-way ANOVA for repeated measures with the factors time/condition and group. Between-group comparisons were performed by using the two-tailed Student's $t$ test. Within-group comparisons were performed by one-way ANOVA for repeated measures, followed by the Dunnett's test for multiple comparisons, using the mean of the four baseline samples as the control.

\section{Results}

Concentrations of catecholamines in dialysates from PFC

Baseline levels of PFC NA and DA were $5.35 \pm 0.19$ and $0.67 \pm 02$ $\mathrm{pg} / 15 \mu \mathrm{l}$ sample. One-way ANOVA indicated no significant differences in NA or DA baseline values among the four groups $\left(F_{(27,76)}=0.6, p=0.93\right.$ and $F_{(27,75)}=0.8, p=0.74$, respectively $)$.

Because stimuli predictive of a food reward are known to be associated with increased DA efflux in the PFC in habituated animals (Bassareo and Di Chiara, 1997; Ahn and Phillips, 1999), we evaluated the contribution of reward-anticipatory responses to PFC NA and DA levels measured during the delayed alternation task. Thus, in the present task (Fig. 1), rats were placed for 10 min (one dialysate sample) in a cage (waiting cage) adjacent to the T-maze before being exposed to the maze for the testing session.

\section{NA}

\section{Conditioned groups}

In rats untrained to alternate in the T-maze, one-way ANOVA for repeated measures indicated a significant effect of time/condition for PFC NA levels $\left(F_{(10,83)}=2.26 ; p<0.05\right)$ (Fig. 3a). Post hoc analysis showed that NA levels measured in the waiting cage did not differ from those in basal conditions (home cage). A small but significant increase $(+16.9 \pm 9.3 \%$; Dunnett's test; $p<0.05)$ in NA efflux occurred when animals visited the maze to locate and eat the food (test condition). NA returned to baseline values when animals were returned to their home cage. In the group of trained rats (i.e., rats that acquired the rule to perform correctly the alternation task), one-way ANOVA for repeated measures indicated a highly significant effect of time/condition $\left(F_{(10,78)}=\right.$ $6.68 ; p<0.0001$ ) (Fig. 3b). As in the untrained group, NA levels measured in the waiting cage were not significantly different from basal conditions. However, in contrast to untrained rats, in the alternation-trained group, performance in the working memory task was associated with a sustained increase in PFC NA efflux $(+62.2 \pm 19.5 \%$; Dunnett's test; $p<0.01)$ lasting for $10 \mathrm{~min}$ after the test $(+41.7 \pm 17.1 \%$; $p<0.01)$. Two-way ANOVA for repeated measures with factors group and time/condition indicated a significant effect of time/condition $(p<0.0001)$, group $(p=0.002)$, and time/condition $\times$ group interaction $(p=$ $0.027)$. We also calculated the area under the curve of NA efflux occurring during the test and in the successive 10 min because it is difficult to exclude that test effects may contribute to NA concentrations in the following sample. Analysis of the overall NA efflux (one-way ANOVA) again revealed a significant difference between trained and untrained animals $\left(F_{(3,32)}=6.42 ; p=\right.$ 0.0016 ) (see Fig. $5 a$ ). Individual comparisons indicated a significant difference between NA efflux of alternation-trained versus that of the untrained group (Student's $t$ test; $p<0.05$ ) and between NA effluxes of trained and their respective basal values $(p<0.001)$ but not between the values of untrained rats and their respective baseline $(p>0.05)$ (see Fig. $5 a)$. Thus, perfor-
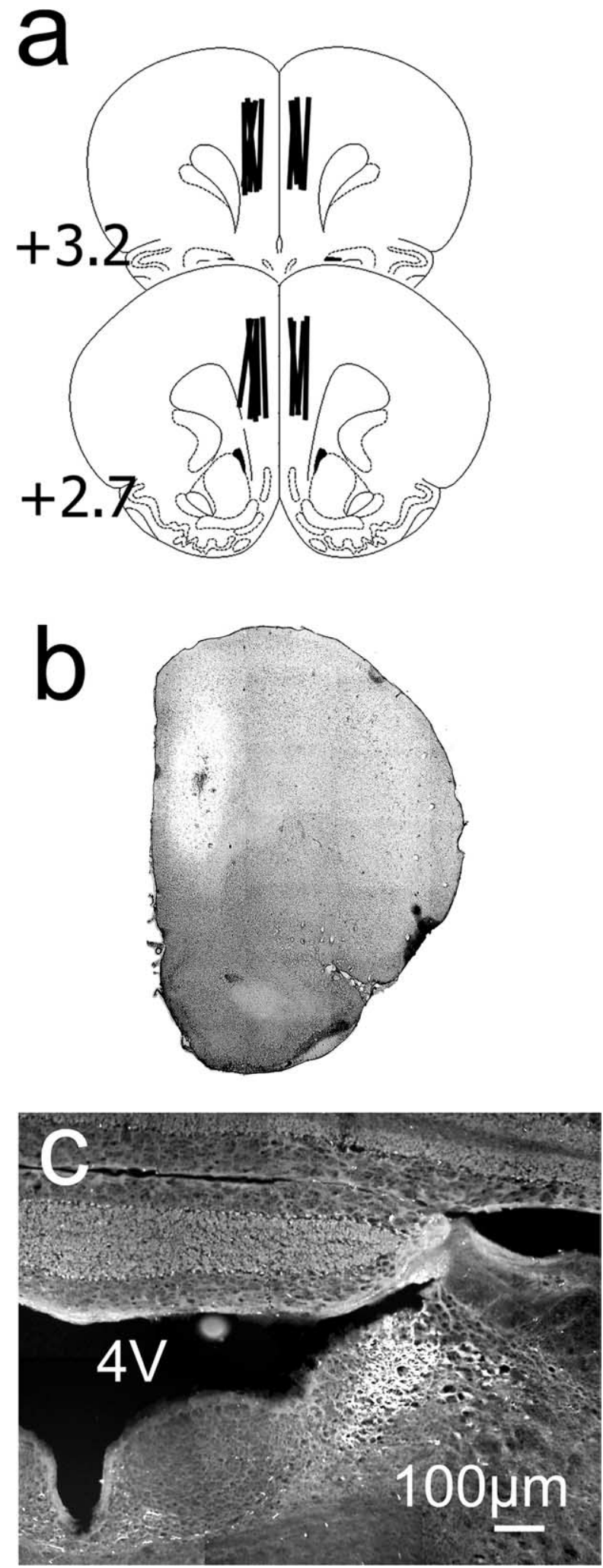

Figure 2. Location of the microdialysis probes in the PFC. $a$, Drawing of coronal sections of the rat brain showing the location of the $4 \mathrm{~mm}$ microdialysis probes (vertical lines). Numbers indicate millimeters from bregma, according to the atlas of Paxinos and Watson (1986). b, Superimposed bright-field/fluorescence image of a $40 \mu \mathrm{m}$ section of PFC showing the diffusion pattern of Fluorogold (brighter area) in the region surrounding the probe. The section is taken from a rat killed $3 \mathrm{~d}$ after the perfusion of the probe for $10 \mathrm{~min}$ with $0.1 \%$ Fluorogold (neutral red staining). $c$, Fluorescence-positive cells in the $L C$ in a section from the same rat in $b$ after perfusion of the fluorescent dye in the PFC. Some Fluorogold-positive neurons are also visible in the caudal portion of the Raphe nucleus. 4V, Fourth ventricle. 

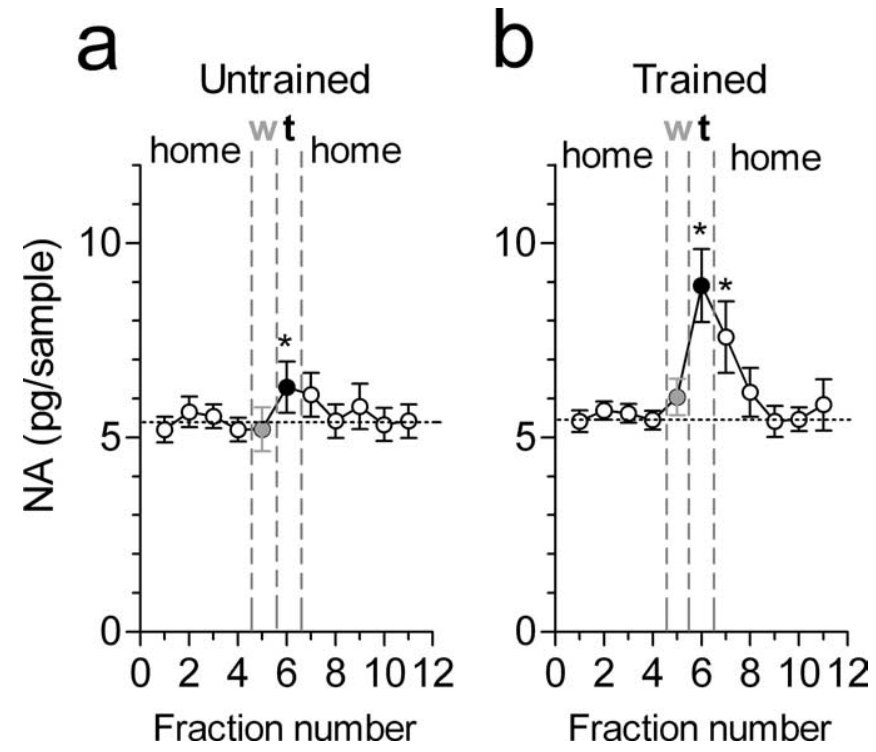

Figure 3. Performance in a delayed alternation task is associated with an increase in NA release in the rat PFC. $a$, Profile of NA release in untrained rats during baseline (white circles), in the waiting cage (w; gray circles), during the task (t; black circles), and again in the home cage (white circles). $b$, Changes in PFC NA levels in rats trained to alternate in the T-maze. Circles are as in $a$. The abscissa represents the number of 10 min dialysate samples. Points represent means \pm SEM of nine animals per group. ${ }^{*} p<0.05$ versus the average of the four baseline values (Dunnett's multiple-comparison test)

mance in a delayed alternation task of working memory is associated with increased PFC NA efflux.

\section{Pseudoconditioned groups}

Similar to the results obtained for the conditioned groups, between-group statistical comparison of the two pseudoconditioned groups by two-way ANOVA for repeated measures revealed a significant group $(p=0.011)$, time/condition $(p=$ $0.015)$, and group $\times$ time/condition interaction $(p=0.049)$ effect. Post hoc analysis revealed a significant difference between NA values in the test condition and those of baseline only for rats trained to alternate in the maze $(+51.70 \pm 20.0 \%$; Dunnett's test; $p<0.05$ ) (see Fig. 6, left). To evaluate the effect of rewardpredictive stimuli on PFC NA levels, comparisons were performed between alternation-trained or -untrained rats and the respective pseudoconditioned groups. In the alternation-trained groups, two-way ANOVA for repeated measures indicated a significant effect of time/condition $(p<0.0001)$ but no effect of treatment $(p=0.58)$ or interaction $(p=0.56)$. Comparison between the pseudoconditioned and the conditioned, alternation-untrained groups did not result in any significant effect of time/condition $(p=0.27)$, treatment $(p=0.63)$, or interaction $(p=0.66)$. Individual comparisons across the different time/conditions between the conditioned and pseudoconditioned groups are shown in Figure 6 (left panels). In both untrained and trained pseudoconditioned groups, there was no significant difference between PFC NA levels in the waiting cage and baseline values (Student's $t$ test) (see Fig. $6 a, b$, left). There was no significant difference in NA values in the waiting cage between conditioned and pseudoconditioned rats either untrained (see Fig. $6 a$, left) or trained to alternate (see Fig. $6 b$, left).

Thus, conditioning had no effect on NA levels in the different time/conditions in rats either trained or untrained to alternate.

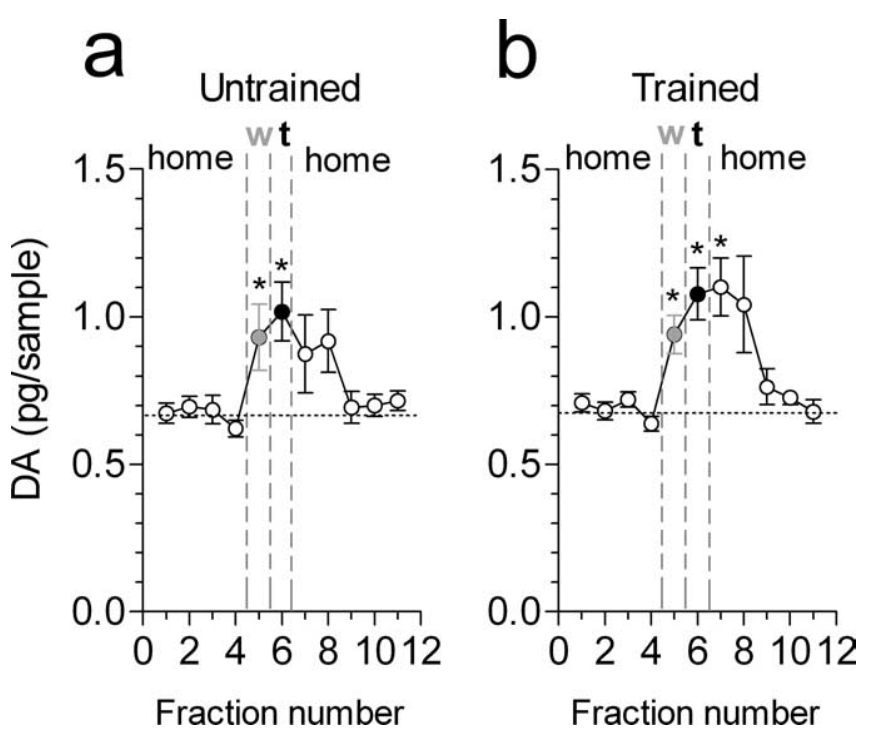

Figure 4. Profile of DA efflux in the rat PFC during the different phases of the behavioral test. $a$, Changes in PFC DA levels in untrained rats during baseline (white circles), in the waiting cage (w; gray circles), during the task (t; black circles), and again in the home cage (white circles). $b$, Changes in PFC DA levels in rats trained to alternate in the T-maze. Circles are as in $a$. The abscissa represents the number of 10 min dialysate samples. Points are means \pm SEM of nine animals per group. ${ }^{*} p<0.05$ versus the average of the four baseline values (Dunnett's multiple-comparison test).

\section{DA}

Conditioned groups

In alternation-untrained rats, one-way ANOVA for repeated measures indicated a significant effect of time/condition $\left(F_{(7,78)}=3.78 ; p<0.001\right)$. Consistent with previous studies showing elevations of PFC DA levels in anticipation of food reward (Bassareo and Di Chiara, 1997; Ahn and Philips, 1999), there was a significant difference between baseline DA levels and those measured in the waiting cage $(+31.8 \pm 5.9 \%$; Dunnett's test; $p<0.01)$. DA efflux remained elevated to similar levels when rats were successively exposed to the maze environment to locate and eat the food $(+37.8 \pm 6.9 \%$; $p<0.01)$. Subsequently, DA levels progressively returned to baseline values when the animals were returned to the home cage (Fig. 4a). A similar pattern of DA changes was also observed in trained animals (Fig. 4b). One-way ANOVA for repeated measures indicated a highly significant effect of time/condition $\left(F_{(7,71)}=7.2 ; p<0.0001\right)$. DA levels in the waiting cage increased to $37.4 \pm 8.5 \%$ of baseline (Dunnett's test; $p<0.05)$, reached $+58.5 \pm 13.0 \%$ of basal values $(p<0.01)$ in the $10 \mathrm{~min}$ sample taken during the alternation test, and remained at such elevated levels for an additional $10 \mathrm{~min}$. Two-way ANOVA for repeated measures with the factors group and time/ condition indicated a significant effect of time/condition $(p<$ $0.0001)$ but not of group $(p=0.06)$ or time/condition $\times$ group interaction $(p=0.34)$. Comparison between the overall DA efflux over $20 \mathrm{~min}$ (area under the curve over the time of the test plus the following 10 min sample) (Fig. $5 b$ ) indicated a significant effect versus average DA efflux in $20 \mathrm{~min}(100 \%)$ for both untrained and trained groups (Student's $t$ test; $p<0.05$ and $p<$ 0.01 , respectively) but no significant difference between DA values during the test in the maze between the alternation-trained and untrained groups ( $p>0.05$ for both) (Fig. $5 b$ ). Thus, the elevations of PFC DA levels in the waiting cage or when animals visited the maze occurred independently of whether the animals were trained or not trained to alternate. 


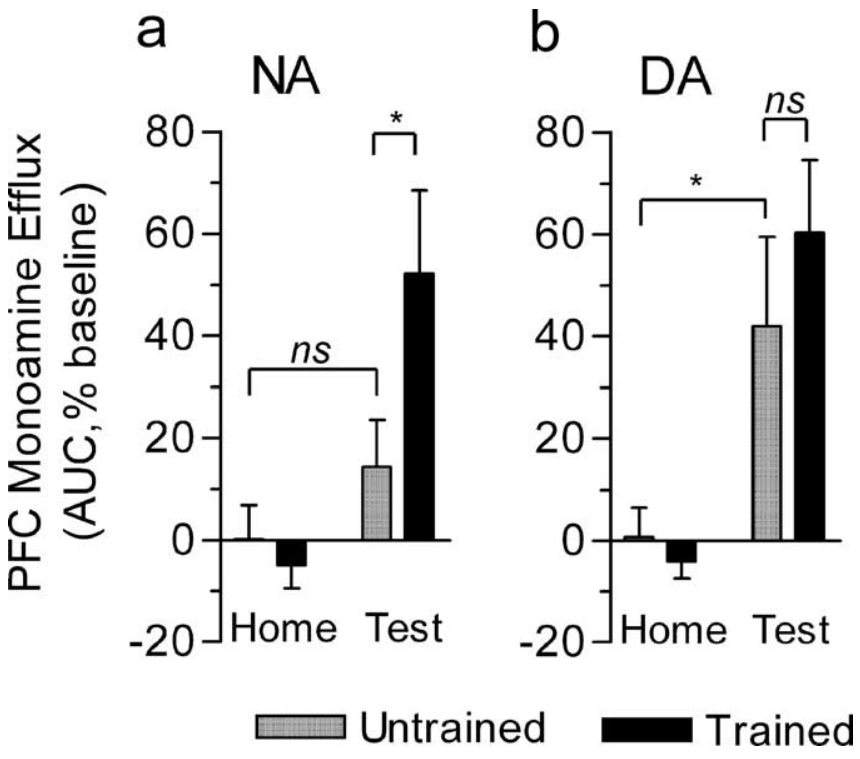

Figure 5. Performance in the alternation task is associated with selective NA efflux in the PFC of trained rats. $a$, Total efflux of NA relative to baseline during the 10 min exposure to the maze and in the successive 10 min sample. $b$, Total efflux of DA during the 10 min exposure to the maze and in the successive $10 \mathrm{~min}$ sample. Values are means \pm SEM of the area under the curve of the monoamine efflux in $20 \mathrm{~min}$ and are normalized with respect to the averaged catecholamine efflux in the home cage in $20 \mathrm{~min}$ (100\%). "Home" bars represent the normalized area of the last two baseline samples ( 20 min efflux) taken before placing the different groups of rats in the waiting cage. ${ }^{*} p<0.5$ (Student's $t$ test). ns, Not significant. Error bars represent SEM.

\section{Pseudoconditioned groups}

As in the case of the conditioned groups, between-group statistical comparison of the two pseudoconditioned groups by two-way ANOVA for repeated measures resulted in a significant effect of time/condition $(p<0.0001)$ but not group $(p=0.42)$ or group $\times$ time/condition interaction $(p=0.64)$. Post hoc analysis revealed a significant difference between DA levels during the test in the maze and those of baseline $(+61.8 \pm 15.5$ and $58.5 \pm$ $13.0 \%$ of baseline for untrained or trained rats, respectively; Dunnett's test; $p<0.001$ for both) but not between the waiting cage and baseline, and this was the case for both groups of pseudoconditioned rats (Fig. 6, right panels). Statistical comparisons between alternation-untrained rats and the respective pseudoconditioned group by two-way ANOVA for repeated measures indicated a significant effect of time/condition $(p<$ $0.0001)$ but no significant treatment or interaction effect. Comparison between alternation-trained rats and the respective pseudoconditioned group indicated a significant time/condition $(p<0.0001)$ and treatment effect $(p=0.02)$ but not of their interaction $(p=0.22)$. Individual comparisons across the different time/conditions between the conditioned and pseudoconditioned groups are shown in Figure 6 (right panels). In both pseudoconditioned groups (untrained or trained to alternate), there was no significant difference between PFC DA levels in the waiting cage and baseline values (Student's $t$ test; $p>0.05$ ) (Fig. $6 a, b$, right). However, there was a significant difference in DA values in the waiting cage between conditioned and pseudoconditioned rats either untrained (Fig. $6 a$, right) or trained to alternate (Fig. $6 b$, right)

Therefore, a double dissociation was observed between PFC NA and DA efflux during performance in the delayed alternation task in a T-maze compared with the control task. NA, but not DA, was activated to a higher level in the delayed alternation task;

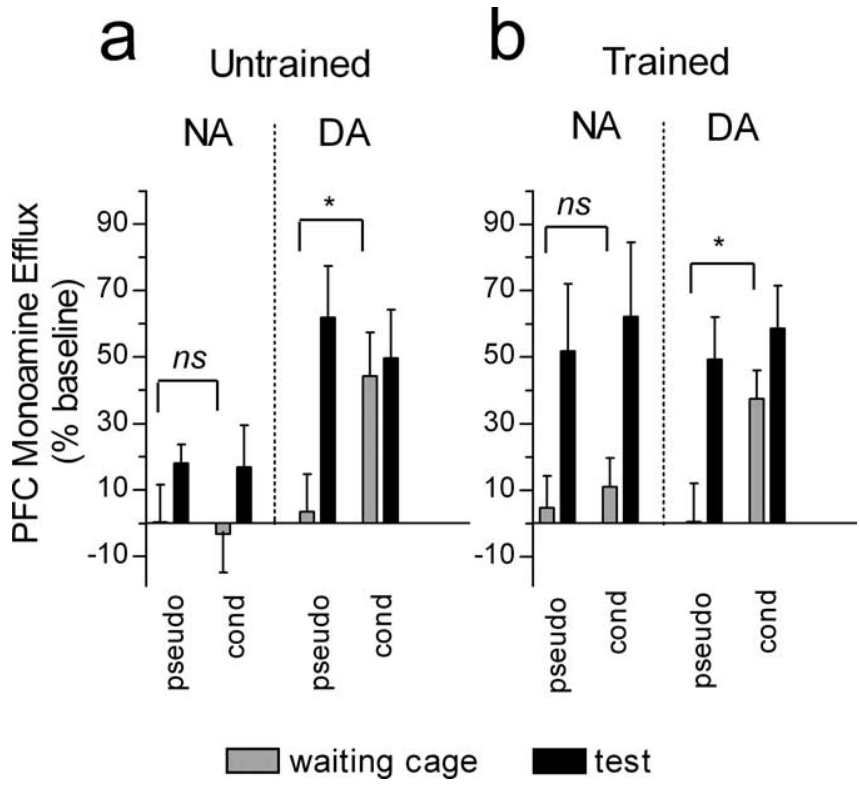

Figure 6. PFC DA, but not NA, efflux increases in the anticipatory phase of the test in conditioned rats. $a$, PFC NA and DA levels during the anticipatory phase of the test (waiting cage) and during the test in the T-maze in rats untrained to alternate. $b$, PFC NA and DA levels during the anticipatory phase of the test (waiting cage) and during the test in the T-maze in rats trained to alternate. Bars represent monoamine levels in 10 min samples, are expressed as the percentage of respective baseline values in each group (average of 4 baseline samples in the home cage), and are means \pm SEM of five [pseudoconditioned groups (pseudo)] or nine [conditioned groups (cond)] animals. * $p<0.05$ (Student's $t$ test). ns, Not significant. Error bars represent SEM.

however, DA, but not NA, was activated during the waiting period before the actual test.

\section{Discussion}

Cortical NA, selective attention, and working memory

These results show that PFC NA efflux increases in rats trained or untrained to alternate and indicate that the LC-noradrenergic system is phasically activated when animals visit the maze environment in search for food. Handling (Dalley and Stanford, 1995; Kawahara et al., 1999; Feenstra et al., 2000) does not contribute to NA elevations because no change in NA efflux was observed when all of the groups of animals were moved from the home cage to the waiting cage or when untrained animals were placed from the maze back to their home cages. Elevations of PFC NA levels in response to stimuli that predict a reward have been shown previously (Mingote et al., 2004). It is known that food restriction or presentation of highly palatable food causes an increase in PFC NA efflux (Cenci et al., 1992; Taber and Fibiger, 1997; Feenstra, 2000; Feenstra et al., 2000). In alternationuntrained animals, we observed moderate elevations of NA levels during the task, but conditioned stimuli signaling reward do not contribute to these levels because no increase in NA efflux was observed in either group when animals were placed in the waiting cage before visiting the maze. We cannot rule out that conditioned NA release may occur during the active phase of the task when rats searched for food in the T-maze, but the marked differences between the procedures of our study and those of Mingote et al. (2004) render a comparison of the results difficult. In contrast, LC neurons are activated by salient stimuli that elicit attentional demands but are not associated with reward (Grant et al., 1988; Aston-Jones et al., 2000). Indeed, the PFC NA innervation is activated by various stimuli, regardless of their rewarding 
or aversive quality (Dalley and Stanford, 1995; Rebec et al., 1997; McQuade et al., 1999; Feenstra, 2000; Feenstra et al., 2000). Therefore, the elevations of NA levels during the active phase of the task in both groups of rats may represent a neurochemical correlate of cortical arousal that is required for the processing of the spatial information for searching and locating food reward; that is, it may reflect a role of LC in attentional processes (AstonJones et al., 2000).

However, the main finding of our work is that the increase in NA levels in animals that were trained to alternate was approximately fourfold larger than that in untrained rats visiting the same test environment. The differential increase in NA levels is neither related to the consumption of a primary reward nor to a difference in the motivational state of the animals because both groups of rats consumed the same amount of the same kind of food during the same time and were equally food restricted. Second, novelty (Dalley and Stanford, 1995; Feenstra, 2000; Feenstra et al., 2000) is unlikely to contribute to the differential PFC NA elevations because both groups were equally habituated to the same testing environment during the acquisition period. Therefore, the difference in NA efflux between rats untrained and trained to alternate indicates a relationship between the attentional demand required by the task difficulty and the phasic LC activity and suggests a specific role of PFC NA in working memory. In the delayed alternation task, trained animals may need to heighten the level of attention to the attended target compared with animals for which the outcome (reward, in the present paradigm) is certain. Thus, we propose that PFC NA elevations represent a correlate of selective attention required for the active maintenance of the rules to achieve a goal (Robbins, 1984; Rainer et al., 1998; Aston-Jones et al., 2000; Everling et al., 2002; Berridge and Waterhouse, 2003).

A theory of PFC function (Miller and Cohen, 2001) postulates that working memory can direct attention via "internal representation of the to-be-attended target object" (Rainer et al., 1998) and identify and process task-relevant stimuli to allow response selection. This "top-down" theory raises the important question of whether the task-related neuronal activity of PFC neurons and the increases in frontal cortical NA release are causally interrelated. In monkeys, pyramidal neurons of the PFC send projections to LC NA neurons (Arnsten and Goldman-Rakic, 1984), and there is evidence in rats for a powerful excitatory input of PFC to LC cells (Jodo and Aston-Jones, 1997; Jodo et al., 1998). PFC neurons fire in response to cues that are being memorized and to stimuli that recall stored information and elicit the behavioral response (Fuster, 1973; Kubota et al., 1974; Niki, 1974). The firing of these neurons is positively correlated with accuracy of memory-guided behavior (Goldman-Rakic, 1995). Moreover, the sustained activity of PFC pyramidal neurons during the delay period of working memory tasks is reduced by noradrenergic antagonists (Li and Mei, 1994; Sawaguchi, 1998; Li et al., 1999). A PFC activity-related representation of the to-be-attended target object may trigger the phasic activation of the LC-noradrenergic system to sustain focus on those targets present in the environment that encode motivational content. Additional electrophysiological studies are needed to assess whether LC neurons are activated during the delay period of working memory tasks.

\section{NA, DA, and PFC function}

Activation of the LC-noradrenergic or the DA corticolimbic systems alone is not sufficient for working memory functions because selective impairment of either NA or DA transmission in the PFC disrupts working memory (Brozoski et al., 1979; Bubser and Schmidt, 1990; Roberts et al., 1994; Arnsten, 1997). Our results show that NA and DA levels undergo moderate, concomitant, and comparably sustained increases during the delayed alternation task in trained animals. Although these neurotransmitters seem to comodulate PFC-dependent working memory, the present results reveal several important differences between DA and NA. First, in contrast to NA, DA elevations that accompanied maze exposure did not differ between trained and untrained rats. Second, levels of DA, but not those of NA, increased in the anticipatory phase of the task. Third, DA elevations during the task, but not those of NA, were not significantly different from those observed in the anticipatory phase of the task, and this was the case for both groups of rats. These results are consistent with microdialysis studies showing that consumption of food or exposure to reward-predicting stimuli consistently increase DA release in the PFC (Bassareo and Di Chiara, 1997; Ahn and Phillips, 1999; Phillips et al., 2004) (but see Mingote et al., 2004). Furthermore, these results correlate with electrophysiological studies in monkeys, indicating that DA neurons fire in response to reward prediction (Schultz, 1998; Schultz and Dickinson, 2000). DA cells show a rate of sustained activity that grows to the expected time of reward and is determined by reward magnitude. The maximal activation of DA neurons occurs under conditions of maximal uncertainty of the outcome (Fiorillo et al., 2003); that is, when $p=50 \%$, as in the present and other tasks of working memory. Thus, the conditions of the present alternation task are the conditions of maximal neuronal DA activation. However, the fact that no significant difference in DA levels was found between trained and untrained rats while animals visited the maze and that pseudoconditioning abolished DA elevations in the waiting cage in both groups seems to indicate that DA release in the PFC primarily encodes information about reward-predicting stimuli, whether or not working memory functions are engaged. This conclusion is consistent with the finding that, during the delay period of a working memory task, DA neurons do not show sustained activity (Schultz et al., 1993). Thus, the role of corticolimbic DA transmission seems to be dissociated from the cognitive functions of working memory. On the other hand, during delayed response tasks, PFC neurons fire at a rate determined by the value of the reward expected at the end of the trial (Watanabe, 1996; Leon and Shadlen, 1999; Watanabe et al., 2002; Roesch and Olson, 2003). Clearly, this prediction of reward implies the elaboration of recalled information that is stored in long-term memory, a process that likely involves hippocampal (Seamans et al., 1998) or other PFC connections. DA release, elicited in response to reward-predictive stimuli and maintained by growing sustained activity to the expected time of reward, may enable taskrelated neuronal activity in the PFC via modulation of its afferent connections. This interpretation implies an indirect role of DA in working memory processes and may explain why elevations of PFC DA levels also occur during working memory tasks after long delays (Phillips et al., 2004). Indeed, it is hard to imagine that the task-relevant information is actively maintained in the PFC over delays of 30 or $60 \mathrm{~min}$ (Phillips et al., 2004). This role of DA in a "gating" mechanism, which has been suggested by neurocomputational models of the dopaminergic modulation of working memory functions (Cohen et al., 1996; Durstewitz et al., 2000; Deco and Rolls, 2003), is also consistent with a top-down PFC control of DA neuronal activity in goal-directed behavior (Miller and Cohen, 2001).

In conclusion, the present results demonstrate that NA efflux in the PFC is selectively increased during a spatial working memory task. In contrast, DA elevations occur independently of 
whether the animals had acquired the trial-specific information necessary for correct task execution. The double dissociation between NA and DA elevations in the PFC observed in the present study support the hypothesis that, in the memory-guided search for food reward, DA is primarily associated with reward expectancy, whereas NA is involved in the selective attention necessary for actively maintaining the rules to achieve the goal.

\section{References}

Ahn S, Phillips AG (1999) Dopaminergic correlates of sensory-specific satiety in the medial prefrontal cortex and the nucleus accumbens of the rat. J Neurosci 22:10958-10965.

Arnsten AF (1997) Catecholamine regulation of the prefrontal cortex. J Psychopharmacol 11:151-162.

Arnsten AF (2001) Modulation of prefrontal cortical-striatal circuits: relevance to therapeutic treatments for Tourette syndrome and attentiondeficit hyperactivity disorder. Adv Neurol 85:333-341.

Arnsten AF, Goldman-Rakic P (1984) Selective prefrontal cortical projections to the region of the locus coeruleus and raphe nucleus in the rhesus monkey. Brain Res 306:9-18.

Arnsten AF, Goldman-Rakic P (1985) Alpha-2 adrenergic mechanisms in prefrontal cortex associated with cognitive decline in aged non-human primates. Science 230:1273-1276.

Arnsten AF, Cai JX, Goldman-Rakic P (1988) The $\alpha$-2 adrenergic agonist guanfacine improves memory performance in aged monkeys without sedative or hypotensive side effects: evidence for $\alpha-2$ receptor subtypes. J Neurosci 8:4287-4298.

Aston-Jones G, Rajkowski J, Cohen J (2000) Locus coeruleus and regulation of behavioral flexibility and attention. Prog Brain Res 126:165-182.

Bassareo V, Di Chiara G (1997) Differential influence of associative and nonassociative learning mechanisms on the responsiveness of prefrontal and accumbal dopamine transmission to food stimuli in rats fed ad libitum. J Neurosci 17:851-861.

Berridge CW, Waterhouse BD (2003) The locus coeruleus-noradrenergic system: modulation of behavioral state and state-dependent cognitive processes. Brain Res Rev 42:33-84.

Brozoski TJ, Brown RM, Rosvold HE, Goldman PS (1979) Cognitive deficit caused by regional depletion of dopamine in prefrontal cortex of rhesus monkey. Science 205:929-932.

Bubser M, Schmidt WJ (1990) 6-Hydroxydopamine lesion of the rat prefrontal cortex increases locomotor activity, impairs acquisition of delayed alternation tasks, but does not affect uninterrupted tasks in the radial maze. Behav Brain Res 37:157-168.

Cai JX, Ma YY, Xu L, Hu XT (1993) Reserpine impairs spatial working memory performance in monkeys: reversal by the alpha-2 adrenergic agonist clonidine. Brain Res 614:191-196.

Cenci MA, Kalèn P, Duan W-A, Björklund A (1992) Regional differences in the regulation of dopamine and noradrenaline release in medial prefrontal cortex, nucleus accumbens and caudate-putamen: a microdialysis study in the rat. Brain Res 581:217-228.

Cohen JD, Braver TS, O'Reilly RC (1996) A computational approach to prefrontal cortex, cognitive control and schizophrenia: recent developments and current challenges. Philos Trans R Soc Lond B Biol Sci 351:1515-1527.

Dalley JW, Stanford SC (1995) Incremental changes in extracellular noradrenaline availability in the frontal cortex induced by naturalistic environmental stimuli: a microdialysis study in the freely moving rat. J Neurochem 65:2644-2651.

Deco G, Rolls ET (2003) Attention and working memory: a dynamical model of neuronal activity in the prefrontal cortex. Eur J Neurosci 18:2374-2390.

Desimone R, Duncan J (1995) Neural mechanisms of selective visual attention. Annu Rev Neurosci 18:193-222.

Durstewitz D, Seamans JK, Sejnowski TJ (2000) Dopamine-mediated stabilization of delay-period activity in a network model of prefrontal cortex. J Neurophysiol 83:1733-1750.

Everling S, Tinsley CJ, Gaffan D, Duncan J (2002) Filtering of neural signals by focused attention in the monkey prefrontal cortex. Nat Neurosci 5:671-676.

Feenstra MGP (2000) Dopamine and noradrenaline release in the prefrontal cortex in relation to unconditioned and conditioned stress and reward. Prog Brain Res 126:133-163.
Feenstra MGP, Botterblom MHA, Mastenbroek S (2000) Dopamine and noradrenaline release in the prefrontal cortex in the light and dark phase: effects of novelty and handling and comparison to the nucleus accumbens. Neuroscience 100:111-117.

Fiorillo CD, Tobler PN, Schultz W (2003) Discrete coding of reward probability and uncertainty by dopamine neurons. Science 299:1898-1902.

Fuster JM (1973) Unit activity in prefrontal cortex during delay-response performance: neuronal correlates of transient memory. J Neurophysiol 36:61-78.

Goldman-Rakic PS (1987) Circuitry of the prefrontal cortex and the regulation of behavior by representational knowledge. In: Handbook of physiology, Vol 5, Higher cortical function (Plum F, Mountcastle VB, eds), pp 373-417. Washington, DC: American Physiology Society.

Goldman-Rakic PS (1995) Cellular basis of working memory. Neuron 14:477-485.

Granon S, Vidal C, Thinus-Blanc C, Changeux J-P, Poucet B (1994) Working memory, response selection and effortful processing in rats with medial prefrontal lesions. Behav Neurosci 5:883-891.

Grant SJ, Aston-Jones G, Redmond DEJ (1988) Responses of primate locus coeruleus neurons to simple and complex sensory stimuli. Brain Res Bull 21:401-410.

Jodo E, Aston-Jones G (1997) Activation of locus coeruleus by prefrontal cortex is mediated by excitatory amino acids input. Brain Res 768:327-332.

Jodo E, Chiang C, Aston-Jones G (1998) Potent excitatory influence of prefrontal cortex activity on noradrenergic locus coeruleus neurons. Neuroscience 83:63-80.

Kawahara Y, Kawahara H, Westerink BH (1999) Comparison of effects of hypotension and handling stress on the release of noradrenaline and dopamine in the locus coeruleus and medial prefrontal cortex of the rat. Naunyn Schmiedebergs Arch Pharmacol 360:42-49.

Kubota K, Iwamoto T, Suzuki H (1974) Visuokinetic activities of primate prefrontal neurons during delayed-response performance. J Neurophysiol 37:1197-1212.

Leon MI, Shadlen MN (1999) Effect of expected reward magnitude on the response of neurons in the dorsolateral prefrontal cortex of the macaque. Neuron 24:415-425.

Li BM, MeiZT (1994) Delayed-response deficit induced by local injection of the alpha2-adrenergic antagonist yohimbine into the dorsolateral prefrontal cortex in young adult monkeys. Behav Neural Biol 62:134-139.

Li BM, Mao ZM, Wang M, Mei ZT (1999) Alpha2-adrenergic modulation of prefrontal cortical neuronal activity related to spatial working memory in monkeys. Neuropsychopharmacology 21:601-610.

McQuade R, Creton, Stanford SC (1999) Effect of novel environmental stimuli on rat behavior and central noradrenaline function measured by in vivo microdialysis. Psychopharmacology 145:393-400.

Miller EK, Cohen JD (2001) An integrative theory of prefrontal cortex function. Annu Rev Neurosci 24:167-202.

Mingote S, de Bruin JPC, Feenstra MGP (2004) Noradrenaline and dopamine efflux in the prefrontal cortex in relation to appetitive classical conditioning. J Neurosci 24:2475-2480.

Niki H (1974) Differential activity of prefrontal units during right and left delayed response trials. Brain Res 70:346-349.

Paxinos G, Watson (1986) The rat brain in stereotaxic coordinates. New York: Academic.

Phillips AG, Ahn S, Floresco SB (2004) Magnitude of dopamine release in medial prefrontal cortex predicts accuracy of memory on a delayed response task. J Neurosci 24:547-553.

Rainer G, Asaad WF, Miller EK (1998) Selective representation of relevant information by neurons in the primate prefrontal cortex. Nature 393:577-579.

Rebec GV, Grabner CP, Johnson M, Pierce RC, Bardo MT (1997) Transient increase in catecholaminergic activity in medial prefrontal cortex and nucleus accumbens shell during novelty. Neuroscience 76:707-714.

Roberts AC, De Salvia MA, Wilkinson LS, Collins P, Muir JL, Everitt BJ, Robbins TW (1994) 6-Hydroxydopamine lesions of the prefrontal cortex in monkeys enhance performance on an analog of the Wisconsin Card Sort Test: possible interactions with subcortical dopamine. J Neurosci 14:2531-2544.

Robbins TW (1984) Cortical noradrenaline, attention and arousal. Psychol Med 14:13-21. 
Roesch MR, Olson CR (2003) Impact of expected reward on neuronal activity in prefrontal cortex, frontal and supplementary eye fields and premotor cortex. J Neurophysiol 90:1766-1789.

Sawaguchi T (1998) Attenuation of delay-period activity of monkey prefrontal neurons by an alpha2-adreneric antagonist during an oculomotor response task. J Neurophysiol 80:2200-2205.

Sawaguchi T, Goldman-Rakic PS (1991) $\mathrm{D}_{1}$ dopamine receptors in prefrontal cortex: involvement in working memory. Science 251:947-950.

Sawaguchi T, Matsumura M, Kubota K (1988) Dopamine enhances the neuronal activity of spatial short-term memory task in the primate prefrontal cortex. Neurosci Res 5:465-473.

Sawaguchi T, Matsumura M, Kubota K (1990) Catecholaminergic effects on neuronal activity related to a delayed response task in monkey prefrontal cortex. J Neurophysiol 63:1385-1400.

Schultz W (1998) Predictive reward signal of dopamine neurons. J Neurophysiol 80:1-27.

Schultz W, Dickinson A (2000) Neuronal coding of prediction errors. Annu Rev Neurosci 23:473-500.

Schultz W, Apicella P, Ljungberg T (1993) Responses of monkey dopamine neurons to reward and conditioned stimuli during successive steps of learning a delayed response task. J Neurosci 13:900-913.

Seamans JK, Floresco SB, Phillips AG (1998) $D_{1}$ receptor modulation of hippocampal-prefrontal cortical circuits integrating spatial memory with executive functions in the rat. J Neurosci 18:1613-1621.
Taber MT, Fibiger HC (1997) Activation of the mesocortical dopamine system by feeding: lack of a selective response to stress. Neuroscience 77:295-298.

Tanila H, Rama P, Carlson S (1996) The effects of prefrontal intracortical microinjections of an alpha-2 agonist, alpha- 2 antagonist and lidocaine on the delayed alternation performance of aged rats. Brain Res Bull 40:117-119.

Wang M, Vijayraghavan S, Goldman-Rakic PS (2004) Selective $\mathrm{D}_{2}$ receptor actions on the functional circuitry of working memory. Science 353:853856.

Watanabe M (1996) Reward expectancy in primate prefrontal cortex. Nature 382:629-632.

Watanabe M, Kodama T, Hikosaka K (1997) Increase of extracellular dopamine in primate prefrontal cortex during a working memory task. J Neurophysiol 78:2795-2798.

Watanabe M, Hirosaka K, Sagakami M, Shirakawa S (2002) Coding and monitoring of motivational context in the primate prefrontal cortex. J Neurosci 22:2391-2400.

Williams GV, Goldman-Rakic PS (1995) Modulation of memory fields by dopamine $\mathrm{D}_{1}$ receptors in prefrontal cortex. Nature 376:572-575.

Zahrt J, Taylor JR, Mathew RG, Arnsten AF (1997) Supranormal stimulation of $\mathrm{D}_{1}$ dopamine receptors in the rodent prefrontal cortex impairs spatial working memory performance. J Neurosci 17:8528-8535. 